\title{
Size Controlled Synthesis of FeCo Alloy Nanoparticles and Study of the Particle Size and Distribution Effects on Magnetic Properties
}

\author{
A. Shokuhfar' ${ }^{1}$ and S. S. S. Afghahi ${ }^{1,2}$ \\ ${ }^{1}$ Advanced Materials and Nanotechnology Research Laboratory, Faculty of Materials Science and Engineering, \\ K.N. Toosi University of Technology, P.O. Box: 19395-1999 Tehran, Iran \\ ${ }^{2}$ Nanotechnology Research Center, Imam Hossein University, 1581618711 Tehran, Iran
}

Correspondence should be addressed to S. S. S. Afghahi; salmanafghahi@gmail.com

Received 8 October 2013; Revised 30 December 2013; Accepted 6 January 2014; Published 24 February 2014

Academic Editor: Luís Cunha

Copyright (C) 2014 A. Shokuhfar and S. S. S. Afghahi. This is an open access article distributed under the Creative Commons Attribution License, which permits unrestricted use, distribution, and reproduction in any medium, provided the original work is properly cited.

\begin{abstract}
In this research the size controlled synthesis of FeCo nanoparticles was done using a quaternary microemulsion system. X-ray diffraction and high resolution transmission electron microscopy of as-synthesized nanoparticles confirm the formation of FeCo alloy nanoparticles. The effects of two process parameters, namely, water to surfactant molar ratio and molar concentration of metal salts, on the size and size distribution of nanoparticles were discussed by the aid of transmission electron microscopy. The size dependency of magnetic properties was also investigated using a room temperature vibrating sample magnetometer. The superparamagnetic-ferromagnetic and single domain-multidomain transition sizes were determined. Then the specific absorption rates at transition sizes were calculated and the best sample for magnetic hyperthermia treatment was introduced.
\end{abstract}

\section{Introduction}

Magnetic nanoparticles are a topic of growing interest because of their versatile applications such as ultrahigh density data storage, drug delivery, magnetic separation and MRI contrast enhancement [1-13]. Among those, magnetic hyperthermia is a novel therapeutic method in which the magnetic nanoparticles are subjected to an alternating magnetic field to generate a specific amount of heat. The generated heat will then raise the temperature of the tumor to about $42^{\circ} \mathrm{C}$ at which certain mechanisms of cell damage are activated [14]. The heat producing mechanisms under A.C. magnetic fields are (1) hysteresis, (2) the Neel or Brownian relaxation, (3) viscous losses [15].

The generated heat is quantitatively described by the specific absorption rate (SAR) of nanoparticles which is related to specific loss per cycle of hysteresis loop $(A)$ by the equation SAR $=A \times f$ where $f$ is the frequency of applied field.
Up to now several models have been proposed to predict the behavior of magnetic nanoparticles under alternating magnetic fields [16]. For superparamagnetic nanoparticles the equilibrium functions are used. The Langevin function $L(\xi)=\operatorname{coth}(\xi)-1 / \xi$ which is valid at zero anisotropy is an example of equilibrium functions where $\xi=$ $\left(\mu_{0} M_{s} V H_{\max }\right) /\left(k_{B} T\right)$ in which $\mu_{0} H_{\max }$ is the external applied field, $M_{s}$ is the spontaneous magnetization of the nanoparticle, $V$ is the volume of nanoparticle, $k_{B}$ is the Boltzmann constant, and $T$ is the temperature. Linear response theory is valid for nanoparticles at superparamagnetic transition size. Based on this theory the area of the hysteresis loop is calculated by $[16]$

$$
A=\frac{\pi \mu_{0}^{2} H_{\max }^{2} M_{s}^{2} V}{3 k_{B} T} \frac{\omega \tau_{R}}{\left(1+\omega^{2} \tau_{R}^{2}\right)}
$$

where $M_{s}$ is the saturation magnetization, $\omega=2 \pi f$, $\tau_{R}=\tau_{N}=\tau_{0} \exp \left(K_{\mathrm{eff}} V /\left(k_{b} T\right)\right)$ is the relaxation time of 
magnetization equal to the Neel relaxation time $\left(\tau_{N}\right)$, and $\tau_{0}$ is the intrawell relaxation time. The Stoner-Wohlfarth model predicts the magnetic response for single domain ferromagnetic nanoparticles. This model neglects thermal activation and assumes a square hysteresis loop which is relevant for $T=0$ or $f \rightarrow \infty$. For magnetic nanoparticles with their easy axes randomly oriented in space the hysteresis area is calculated by [16]:

$$
A=2 \mu_{0} H_{c} M_{s}=1.92 K_{\text {eff }},
$$

where $\mu_{0} H_{c}$ is the coercive field and $K_{\text {eff }}$ is the effective uniaxial anisotropy of the nanoparticle. The key factor to obtain the maximum SAR in the conventional clinical hyperthermia treatments $\left(f=100 \mathrm{kHz}, \mu_{0} H_{\max }=20 \mathrm{mT}\right.$, and $\left.T=300 \mathrm{~K}\right)$ is the anisotropy of nanoparticles.

Calculations of SAR as a function of anisotropy in the above-mentioned size regimes reveal that the maximal SAR would be obtained at single domain-multidomain transition size. So producing nanoparticles in this range for use in hyperthermia treatment is of high value from technical and clinical aspects.

FeCo alloy has the highest saturation magnetization among all binary magnetic alloys [1]. Several methods have been used to synthesize FeCo alloy nanoparticles which include arc discharge [2], polyol [3-7], hydrothermal [8], reaction under autogenic pressure at elevated temperature (RAPET) [9], thermal decomposition [10], wet chemical $[11,12]$, and coprecipitation $[13,17,18]$. The morphology and size distribution of as-synthesized nanoparticles are not well controlled in most of these processes. To obtain the best properties for magnetic hyperthermia treatments the size distribution is an effective parameter. Researches show the loss of SAR due to nanoparticle size distributions. So employing a method capable of producing monodisperse nanoparticles is of high value.

Microemulsion technique is a method capable of controlling the shape, size, and size distribution of nanoparticles [19]. In this process nanoparticles precipitate inside micelles. The micelle is in the form of sphere of oil in water (normal micelle) or water in oil (reverse micelle) which is surrounded by a layer of surfactant molecules [20]. The technique could be used to synthesize mineral [21] or organic compounds [22].

There are few works on the synthesis of FeCo alloy nanoparticles. The novel quaternary system of water/cetyltrimethylammonium bromide (CTAB)/1-butanol/isooctane was employed for synthesis of FeCo alloy nanoparticles which has not been used before. Unlike other synthetic methods the proposed route is capable of controlling the size of nanoparticles in the range of $1-10 \mathrm{~nm}$. This is achieved by controlling the water to surfactant molar ratio $(R)$ and molar concentration of metal salts. This capability is of vital importance for investigating the heating effect of magnetic nanoparticles under A.C. magnetic fields.

In the present research, the shape and size controlled synthesis of iron cobalt alloy nanoparticles was carried out in the reverse micelles of water in isooctane and the magnetic properties of as-synthesized nanoparticles were studied to investigate their potential usefulness in magnetic hyperthermia treatment.

\section{Materials and Methods}

Iron (III) chloride hexahydrate $\left(\mathrm{FeCl}_{3} \cdot 6 \mathrm{H}_{2} \mathrm{O}(\% 99+)\right)$, isooctane, 1-butanol, sodium borohydride $\left(\mathrm{NaBH}_{4}(\% 99+)\right)$, and cetyltrimethylammonium bromide (CTAB) were purchased from MERCK chemicals and used as received with no further purification. Cobalt acetate tetrahydrate $\left(\mathrm{Co}\left(\mathrm{CH}_{3} \mathrm{COO}\right)_{2} \cdot 4 \mathrm{H}_{2} \mathrm{O}(\% 99+)\right)$ was supplied by MP Biomedicals. High purity nitrogen gas (\%99.99+) was used to provide an oxygen-free environment during the synthesis procedure.

The key to formation of a microemulsion is the formation of a transparent and thermodynamically stable solution which forms at certain ratios of aqueous phase/surfactant/oil phase. Microemulsion 1 (ME1) and microemulsion 2 (ME2) were prepared on the basis of quaternary phase diagram of water/CTAB/1-butanol/isooctane which is described elsewhere [23].

$\mathrm{Fe}_{0.65} \mathrm{Co}_{0.35}$ alloy nanoparticles were prepared by mixing equal volumes of ME1 and ME2 containing metal salts and precipitating agent, respectively. The $\left[\mathrm{NaBH}_{4}\right] /[$ metal salts $]$ molar ratio was kept at 2 to ensure that all of precursors are reduced to zerovalent metal. First ME1 was transferred into a three-necked round bottom flask and then ME2 was added using a dropping funnel to vigorous stirring ME1 under $\mathrm{N}_{2}$ atmosphere. Black precipitates of FeCo alloy nanoparticles appeared immediately after mixing of the two microemulsions.

After 10 minutes of reaction the solution was centrifuged and washed with chloroform, ethanol, and acetone several times to remove all residual elements. Some of as-synthesized powders were annealed in a tube furnace at $350^{\circ} \mathrm{C}$ and $550^{\circ} \mathrm{C}$ for 20 minutes under $\mathrm{H}_{2}$ atmosphere.

Characterization of samples was done using X-ray diffraction (XRD) (PANalytical X'Pert Pro MPD with $\mathrm{Cu}$ $k_{\alpha}$-radiation), scanning transmission electron microscope (STEM) (ZEISS EM10-C at $100 \mathrm{KV})$, and high resolution transmission electron microscope (HRTEM) (JEOL JEM2100 at $200 \mathrm{KV}$ ). Elemental analysis was done using an energy dispersive X-ray spectroscopy (EDS) detector attached to the HRTEM. The magnetic properties of samples were analyzed using a room temperature $(300 \mathrm{~K})$ vibrating sample magnetometer. The samples and process conditions are summarized in Table 1.

\section{Results and Discussion}

3.1. Microstructural Characterization. Figure 1 shows XRD patterns for as-synthesized W3 and annealed samples. As seen from Figure 1(a) there is no major peak. Nanoparticle approximate size could be derived from the Scherrer formula $(d=0.94 \lambda /(\beta \cos \theta))$ based on the $\mathrm{Cu}_{k \alpha}$ radiation and the FWHM of the peak. But for very small sizes $(<5 \mathrm{~nm})$ by considering the drastic peak widening and intensity decreasing there would not be any distinguishable peak. Also 


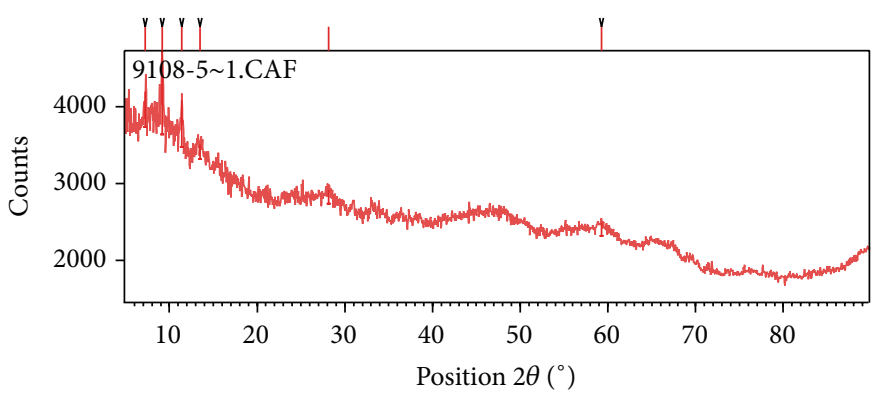

(a)

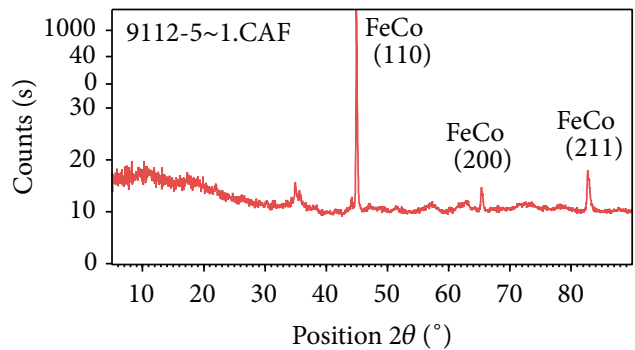

(b)

FIGURE 1: XRD patterns of W3 sample in (a) as synthesized state and (b) annealed at $550^{\circ} \mathrm{C}$ for 20 minutes.

TABLE 1: Samples and process conditions.

\begin{tabular}{lcc}
\hline Sample & $\begin{array}{c}\text { Water/surfactant } \\
\text { molar ratio }(R)\end{array}$ & $\begin{array}{c}\text { Molar concentration } \\
\text { of metal salts }\end{array}$ \\
\hline W1 & 6 & $1 \mathrm{M}$ \\
W2 & 8 & $1 \mathrm{M}$ \\
W3 & 12 & $1 \mathrm{M}$ \\
W4 & 37 & $1 \mathrm{M}$ \\
M1 & 12 & $0.5 \mathrm{M}$ \\
M2 & 12 & $1.5 \mathrm{M}$ \\
M3 & 12 & $2 \mathrm{M}$ \\
\hline
\end{tabular}

the bad crystallinity of nanoparticles (Figure 2(b)) as a result of fast borohydride reduction fortifies this problem.

Figure 1(b) shows the diffraction pattern of W3 sample after annealing at $550^{\circ} \mathrm{C}$ for 20 minutes under $\mathrm{H}_{2}$ atmosphere. XRD patterns reveal the formation of $\alpha$-bcc structured FeCo alloy (at $2 \theta=44.83^{\circ}, 65.32^{\circ}$ and $84^{\circ}$ ). These values agree with JCPDS file for FeCo. Also a small quantity of $\mathrm{CoFe}_{2} \mathrm{O}_{4}$ (at $2 \theta=35.4^{\circ}, 62.4^{\circ}$ ) is observed due to partial oxidation of the sample by exposing to air after annealing procedure. It has been found that FeCo is a substitutional alloy with a bcc structure from pure Fe to about 80 at. \% Co and fcc for 90 at. $\%$ Co [18]. This agrees well with our result.

Figure 2 shows conventional (Figure 2(a)) and high resolution (Figure 2(b)) TEM images of W3 sample. Electron diffraction pattern (inset of Figure 2(a)) and HRTEM image also confirm the formation of bcc structured iron cobalt alloy. EDS analysis shows Fe and Co peaks in which the Fe peak is sharper than that of Co indicating higher content of Fe. $\mathrm{Cl}$ peak is from the residual chloroform which was used to wash as-synthesized nanoparticles. Also an oxygen peak is observed due to the partial oxidation of FeCo.

Figure 3 shows the effect of water to surfactant molar ratio $(R)$ on the morphology, size and size distribution of as-synthesized nanoparticles. The mean size, and size distribution of each specimen were determined by inspecting about 50 TEM micrographs. It is evident that all samples have spherical shape due to the nature of the used surfactant and cosurfactant. Cetyltrimethylammonium bromide (CTAB) which has been used by Schulman for the first time (Hoar and Schulman, 1943) has a hydrophilic head and a lipophilic tail which makes it soluble in both polar and nonpolar solvents. In this quaternary system the polar cosurfactant (1-butanol) makes ion-dipole interaction with the surfactant and forms spherical aggregates in which the polar (ionic) ends of the surfactant molecules are oriented towards the center. We observed that without the addition of 1-butanol the transparent microemulsion would not form. In fact the role of 1-butanol is to act as an electronegative spacer which minimizes the repulsive forces between polar heads of $\mathrm{CTAB}$ molecules and lets them be aggregated in the form of spherical micelles.

3.2. Effect of Water to Surfactant Molar Ratio. Figure 3 indicates the increasing of the mean size of nanoparticles with $R$. As the $R$ value decreases, the relative amount of water reduces and a smaller micelle would be obtained. Therefore the limiting stability of nanoreactors increases leading to smaller nanoparticles. Also it was observed that the spherical shape of nanoparticles would not be affected by changing the $R$ value unlike surfactants like polyvinylpyrrolidone (PVP) [24].

The $\mathrm{W}$ series of samples evidences very narrow (about $3 \mathrm{~nm}$ ) size distribution which is related to the nature of the surfactant. In fact the surfactant has a double influence on the particle formation process: (1) particle stabilization and (2) growth control. The $R$ value affects the former by determining the micellar core size, but the latter is influenced by the nature of the surfactant. The growth mechanism in microemulsions is based on intermicellar exchange [25]. A rigid surfactant surface layer tends to resist opening, thus the reaction is slowed down and simultaneous nucleation and growth occur. This in turn results in the formation of large nanoparticles with broad size distribution. But CTAB provides a very flexible film [25] which facilitates the coalescence exchange between micellar cores. The high exchange rate leads to a uniform nucleation and growth resulting in a narrow size distribution. As noted by Carrey et al., a broad size distribution decreases the maximum achievable SAR [16]. Therefore for as-synthesized FeCo nanoparticles the negative effect of a broad size distribution is not expected.

3.3. Effect of Molar Concentration of Metal Precursors. Figure 4 demonstrates the effect of molar concentration of 


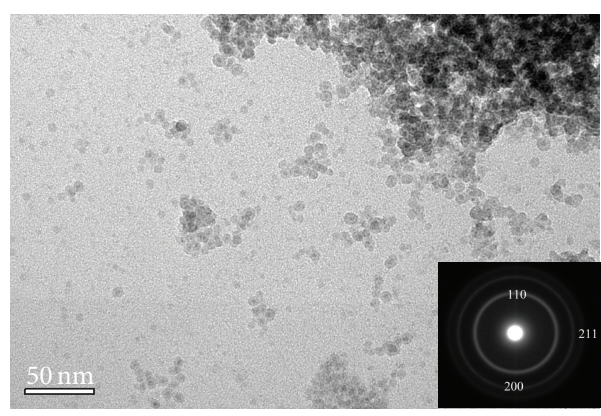

(a)

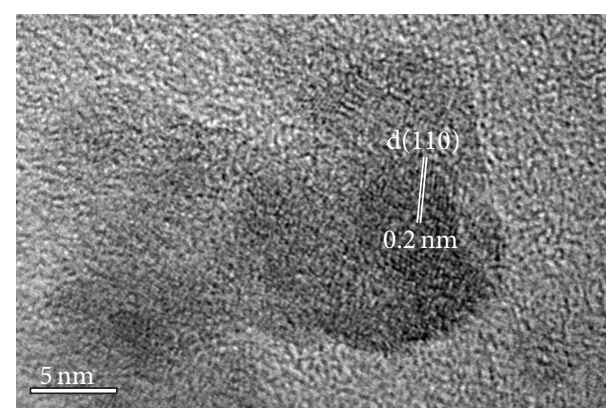

(b)

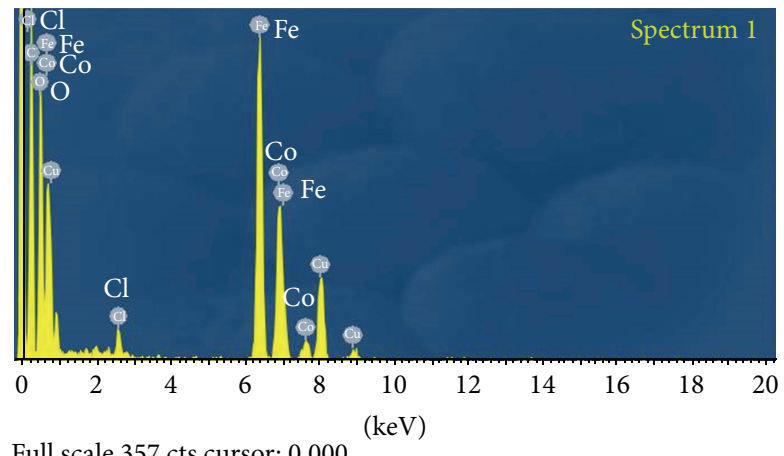

(c)

FIGURE 2: (a) TEM micrograph of W3 sample (inset: selected area diffraction pattern). (b) High resolution TEM image of W3 sample. (c) EDS spectra of W3 sample showing $\mathrm{Fe}, \mathrm{Co}, \mathrm{Cl}$, and $\mathrm{O}$ peaks.

metal salts on the size and size distribution of nanoparticles. It is evident that increasing the molar concentration of metal precursors inversely affects the nanoparticle size. Since the borohydride reduction of metal precursors is almost instantaneous, a huge number of nuclei will form at the first stage of process followed by the nanoparticle growth via intermicellar exchange. Since at high concentrations of metal salts the supersaturation factor $\lambda(t)$ is higher, according to the Adamson equation for homogeneous nucleation, the nucleation rate is higher [26]:

$$
k_{n}(i, t)= \begin{cases}0, & i<n^{*}, \\ i k_{0} \exp \left(\frac{-16 \pi \sigma^{2} v_{m}^{2}}{3\left(k_{b} T\right)^{2}(\ln \lambda(t))^{2}}\right), & i \geq n^{*},\end{cases}
$$

where $\sigma$ is the interfacial tension between solid nucleus and surrounding drop liquid, $v_{m}$ is the volume of one precipitate molecule, $\lambda$ is the supersaturation ratio of liquid product molecules, and $n^{*}$ is the critical number of liquid product molecules in a nucleus. Thus at higher concentrations of metal precursors the number of stable nuclei is higher and consequently the number of remaining product molecules is smaller. Therefore the growth of nanoparticles which proceeds by the addition of product molecules is slowed down and the terminal nanoparticle size reduces. Similarly at lower concentrations of metal precursors the number of formed nuclei is lower providing more product molecules to contribute in the growth stage leading to larger terminal nanoparticles.
3.4. Magnetic Studies. Magnetization curves for $\mathrm{W}$ and $\mathrm{M}$ series of samples are outlined in Figures 5(a) and 5(b). $M_{s}$ and $H_{c}$ values are seen to be size dependent. As for W1 sample with mean size of $2 \mathrm{~nm}$, the $M_{s}$ equals 8 (emu/g). For W2, $\mathrm{W} 3$, and W4 (with mean sizes of $2.5 \mathrm{~nm}, 4 \mathrm{~nm}$, and $9 \mathrm{~nm}$ ) the $M_{s}$ values reach 22, 36 and 65 (emu/g) respectively. This is also observed for M1, M2, and M3 with sizes of $6 \mathrm{~nm}, 3 \mathrm{~nm}$, and $1.5 \mathrm{~nm}$, and corresponding $M_{s}$ of 49,23 , and 6 (emu/g). Also as expected some of the particles (W1, W2, M1, and M2) show superparamagnetic behavior with zero coercivity. In ferromagnetic metals like $\mathrm{Fe}, \mathrm{Co}$, and $\mathrm{Ni}$ the exchange interaction is positive, favoring the parallel alignment of spins. But when the particle size decreases, the majority of atoms and consequently spins are located at the nanoparticle surface. Regardless of the lower spin density at the surface, the structural changes at the surface should be considered. It is proved that the average lattice parameter in nanoparticles is less than their corresponding bulk materials mainly due to the bond length reduction [27].

This bond length contraction induces the overlapping of atomic orbitals, which reduces the atomic dipole moment. Also due to the lower coordination number for surface atoms the exchange coupling between dipoles is less than internal atoms and therefore the magnetic moments tend to fluctuate. The sum of these effects disorder spins at the surface to form a magnetic dead layer. The effect of this layer is reducing the total magnetic moment of nanoparticle [28]. This event is important mainly at sizes below $5 \mathrm{~nm}$ in which about $\% 50$ of total atoms are located at the surface. By increasing the 

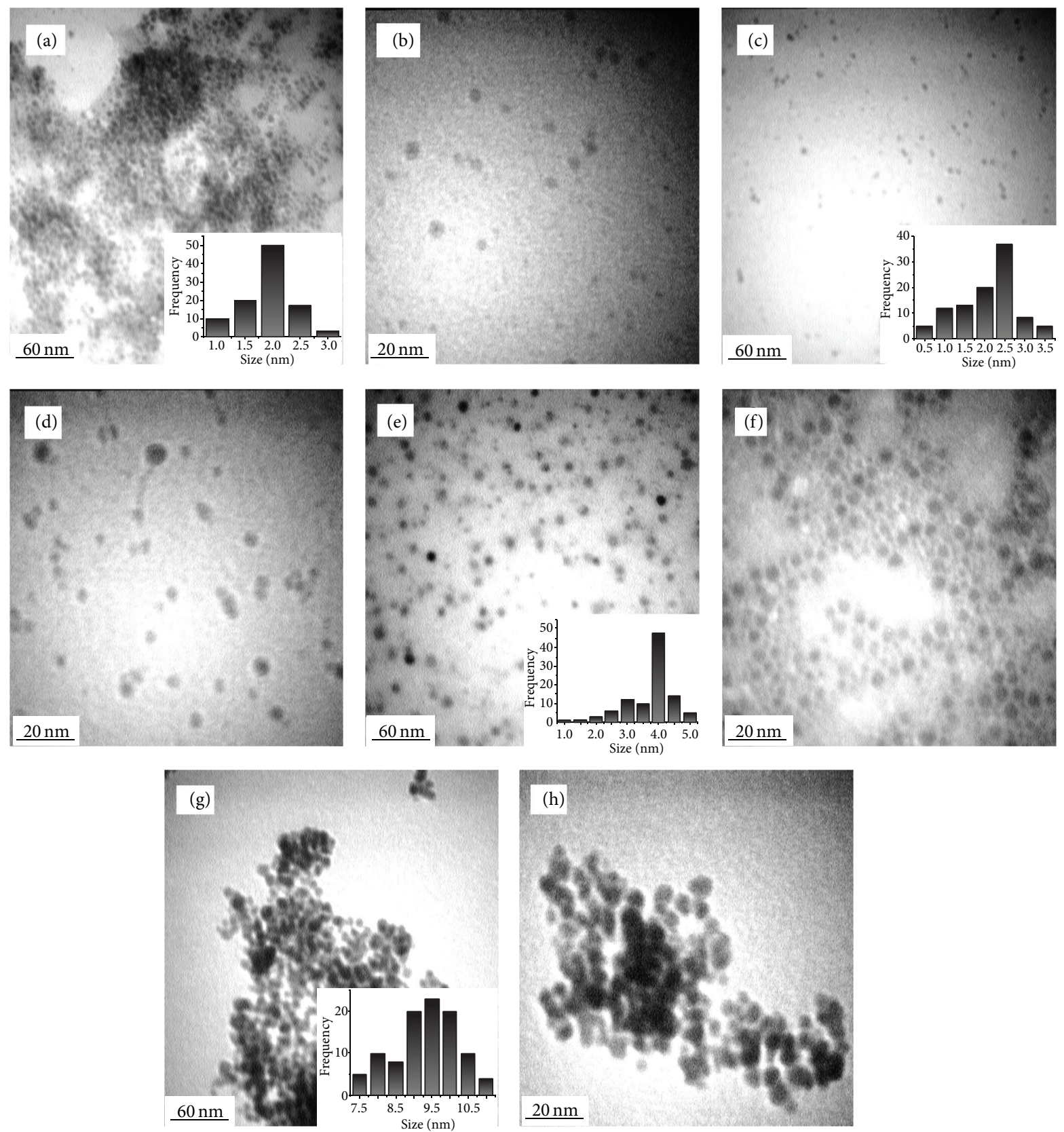

FIGURE 3: TEM micrographs of as-synthesized nanoparticles and corresponding size distributions: (a) W1, (b) W1 at higher magnification, (c) W2, (d) W2 at higher magnification, (e) W3, (f) W3 at higher magnification, (g) W4, and (h) W4 at higher magnification.

nanoparticle size the thickness of this dead layer reduces and the magnetization of nanoparticle increases [28].

It is also seen from Figures 5(a) and 5(b) that the coercivity is size dependent. In fact by increasing the nanoparticles size the coercivity increases such that for $\mathrm{W} 4$ sample the high coercive field of 100 Oe is achieved. The reduced coercive force in terms of nanoparticle size at constant temperature is described as

$$
h_{c}=1-\left(\frac{V_{p}}{V}\right)^{1 / 2}=1-\left(\frac{D_{p}}{D}\right)^{3 / 2}
$$

where $h_{c}$ is the reduced coercive field $\left(H_{c} / H_{c,(T=0)}\right), D$ is the nanoparticle size, and $D_{p}$ is the critical nanoparticle size in which the anisotropy energy (KV) dominates the thermal energy $\left(k_{b} T\right)$ and the magnetic properties change from superparamagnetic to ferromagnetic.

The critical size $D_{p}$ depends on the composition of nanoparticles as for iron oxide nanoparticles and it has the value of $12 \mathrm{~nm}$ [29] or for $\mathrm{Fe}_{74.5-x} \mathrm{Cu}_{x} \mathrm{Nb}_{3} \mathrm{Si}_{22.5-y} \mathrm{~B}_{y}$ alloy nanoparticles $D_{p}$ is dependent on $x$ and $y$ and changes from $10 \mathrm{~nm}$ for 1 at $\% \mathrm{Cu}$ and 9 at $\% \mathrm{~B}$ to $15 \mathrm{~nm}$ for 1 at $\% \mathrm{Cu}$ and 6 at $\%$ B [30]. When $D \leq D_{p}$, the coercive field is zero and further increasing in nanoparticle size beyond $D_{p}$ increases the coercivity. It is why for both $\mathrm{W}$ and $\mathrm{M}$ series of samples 

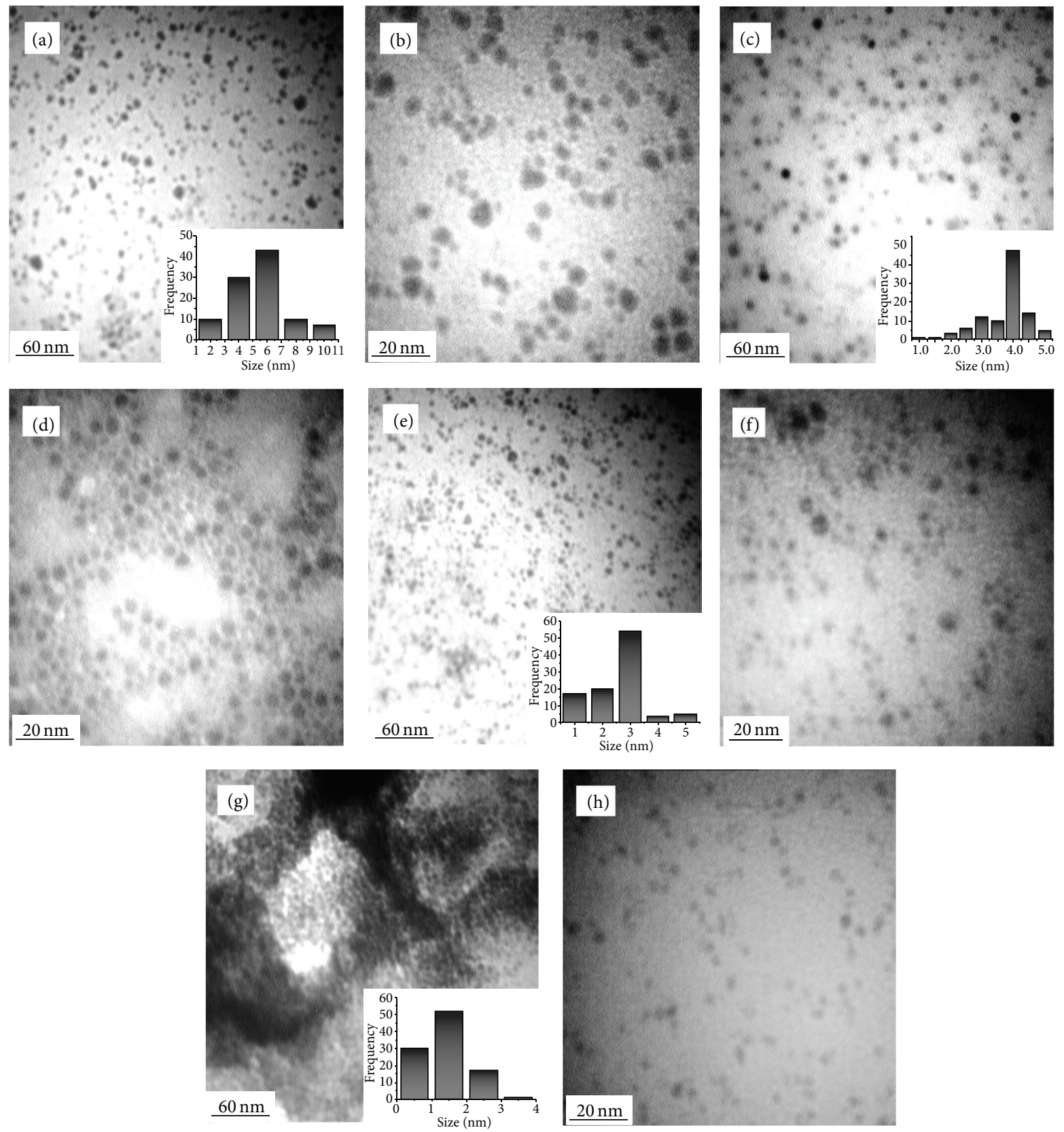

FIGURE 4: TEM micrographs of as-synthesized nanoparticles and corresponding size distributions: (a) M1, (b) M1 at higher magnification, (c) W3, (d) W3 at higher magnification, (e) M2, (f) M2 at higher magnification, (g) M3, and (h) M3 at higher magnification.

the coercivity increases with size. Only an exception is observed in the case of M3 specimen in which regardless of its greater size the coercivity is less than W3 sample. The reason could be the partial oxidation of M3 sample, but more investigations are needed.

Figure 6 shows TEM images and corresponding hysteresis curves for W3 annealed samples. It is seen from Figures 6(a) and 6(b) that the nanoparticles have grown up by fusionfission to a mean size of 60 and $25 \mathrm{~nm}$, respectively. As expected, by increasing the size of nanoparticles the corresponding saturation magnetizations have been increased (to $128 \mathrm{emu} / \mathrm{g}$ and $78 \mathrm{emu} / \mathrm{g}$ ) but are still smaller than the bulk value $(240 \mathrm{emu} / \mathrm{g})$.

Figure 7 shows the coercivity as a function of particle size. It is seen that the coercivity has been decreased from 100 Oe for as-synthesized W3 to 60 and 40 Oe for annealed samples. The reason lies in the mechanism of magnetization. In multidomain particles magnetization reversal takes place by the motion of domain walls, but in single domain particles the magnetization reversal occurs by coherent reversal of the magnetic moment which requires the anisotropy energy to be dominated. Since the anisotropy energy is much higher 


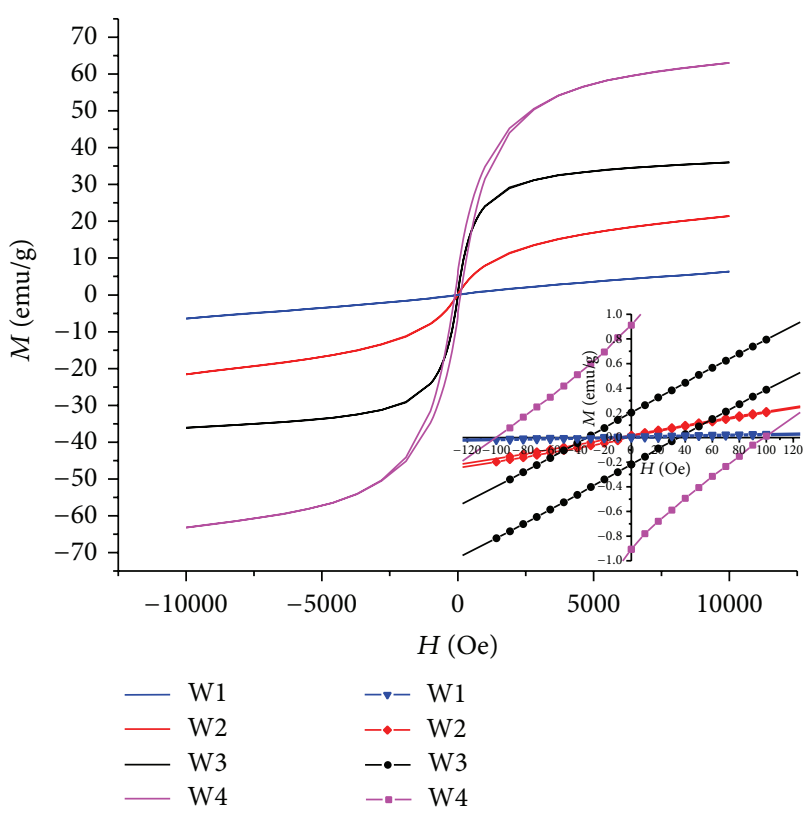

(a)

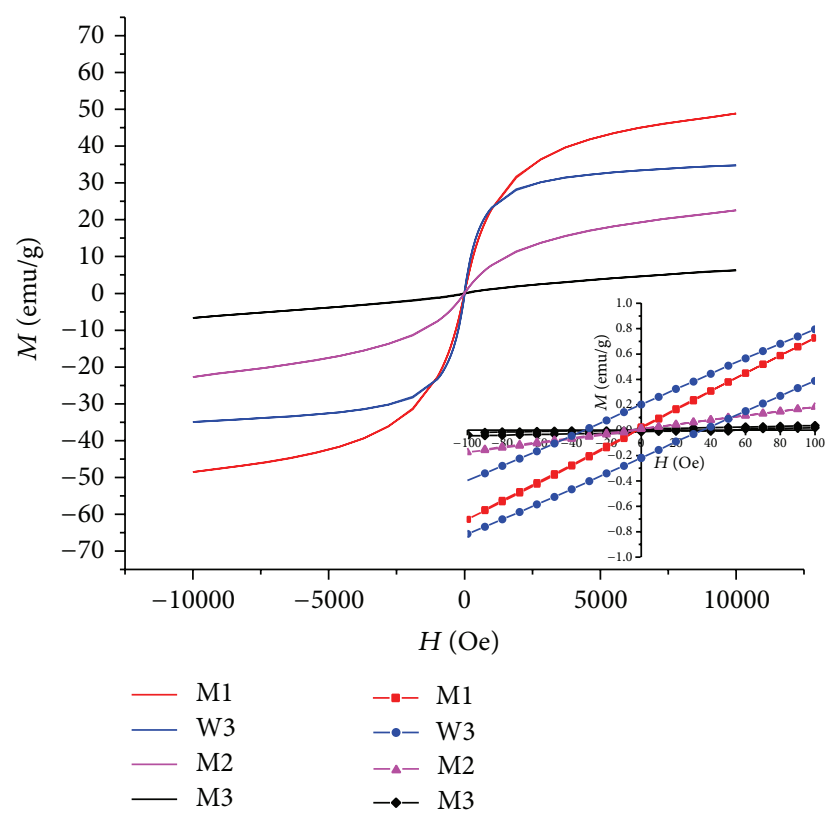

(b)

FIGURE 5: Magnetization curves for as-synthesized nanoparticles showing the effect of (a) water to surfactant molar ratio (R) (b) concentration of metal salts.

than domain wall energy, the coercivity increases by the transition from multi-domain to single domain size regime. But with further decreasing the size, the coercivity diminishes as the result of decreasing the anisotropy energy (KV) such that below $D=4 \mathrm{~nm}$ the coercivity reaches zero exhibiting superparamagnetic behavior. Therefore $D_{p}$ for assynthesized FeCo nanoparticles is about $4 \mathrm{~nm}$. So it is inferred that the transition from superparamagnetic to ferromagnetic is at $4 \mathrm{~nm}$ and the transition between single domain-multi domain size regimes is at $9 \mathrm{~nm}$.

3.5. Calculation of SAR. Some researches on the inductive magnetic properties of nanoparticles represent the superparamagnetic-ferromagnetic transition to have maximum SAR [15] and some other works show that the maximum SAR is achieved at single domain-multi domain boundary [16]. For single domain-multi domain transition size the Stoner-Wohlfarth model is used to estimate the magnetic response. Therefore for W4 sample on the basis of (5) for random orientation nanoparticles we have

$$
2 \mu_{0} H_{c,(T=0 \mathrm{~K})} M_{s}=1.92 K_{\mathrm{eff}},
$$

where $H_{c,(T=0 \mathrm{~K})}$ is the coercive field at $T=0 \mathrm{~K}$ which could be calculated from $H_{c,(T=300)}$ using the following equation [16]:

$$
\frac{H_{c,(T=0 \mathrm{~K})}}{H_{c,(T=300 \mathrm{~K})}}=\frac{1-\left(\left(k_{B} T_{1} / K_{\mathrm{eff}} V\right)\left(\ln \left(1 / f \tau_{0}\right)\right)\right)^{3 / 4}}{1-\left(\left(k_{B} T_{2} / K_{\mathrm{eff}} V\right)\left(\ln \left(1 / f \tau_{0}\right)\right)\right)^{3 / 4}}
$$

where $k_{B}$ is the Boltzmann constant, $V$ is the volume of nanoparticle, $f$ is the measurement frequency $5.5 \times 10^{-4}$ $(\mathrm{Hz}), \tau_{0}=10^{-10}(\mathrm{~s}), T_{1}=0 \mathrm{~K}$, and $T_{2}=300 \mathrm{~K}$. By solving the system of (5) and (6) simultaneously, the effective anisotropy would be calculated as $K_{\text {eff }}=3.8 \times 10^{4}\left(\mathrm{~J} / \mathrm{m}^{3}\right)$ and $\mu_{0} H_{c,(T=0 \mathrm{~K})} \approx 145 \mathrm{mT}$. Then the coercive field at $T=300 \mathrm{~K}$ and the frequency of hyperthermia treatment $f=100 \mathrm{kHz}$ could be calculated from (7) [16]:

$$
\mu_{0} H_{c,\left(T_{1}, f\right)}=0.48 \mu_{0} H_{k}\left[1-\left(\frac{k_{B} T_{1}}{K_{\mathrm{eff}} V}\left(\ln \frac{1}{f \tau_{0}}\right)\right)^{3 / 4}\right] .
$$

Lacroix et al. [31] have shown that the results from the StonerWohlfarth model for random orientation FeCo nanoparticles are coherent with experimental results from calorimetry. According to equation $\mu_{0} H_{(c, T=0)}=0.48 \mu_{0} H_{K}$ at $T=0 \mathrm{~K}$ and (7) at $T=300 \mathrm{~K}$ and $f=100 \mathrm{KHz}$ for the Stoner-Wohlfarth nanoparticles $\left(A \cong 2 \mu_{0} H_{c} M_{s}\right.$ ) it could be calculated that $A \cong 8.47(\mathrm{~mJ} / \mathrm{g})$ and SAR $=847(\mathrm{w} / \mathrm{g})$.

For the above calculations to be valid the condition $\mu_{0} H_{\text {max }} \geq \mu_{0} H_{c,(T=300 \mathrm{~K}, f=100 \mathrm{KHz})} \approx 70 \mathrm{mT}$ should be satisfied such that in each reversal of the applied field the full area of the hysteresis loop could be placed within the limits of the applied alternating field.

The obtained value of SAR is comparable to the highest value of $1700(\mathrm{~W} / \mathrm{g})$ reported by Mehdaoui et al. for iron based nanoparticles [32] and larger than values of $450(\mathrm{~W} / \mathrm{g})$ and 198 $(\mathrm{W} / \mathrm{g})$ for magnetite nanoparticles reported by Bakoglidis et al. [29] and Wang et al. [33], respectively. It should be noted that a broad range of SAR was calculated by Carrey et al. [16] depending on the anisotropy of the nanoparticles ranging from 1 to $2000(\mathrm{~W} / \mathrm{g})$.

For superparamagnetic-ferromagnetic transition the LRT approximation should be applied. For the sake of simplicity it is assumed that $K_{\text {eff }}=3.8 \times 10^{4}\left(\mathrm{~J} / \mathrm{m}^{3}\right)$. Considering $\tau_{R}=\tau_{N}=$ $\tau_{0} \exp \left(K_{\text {eff }} V /\left(k_{b} T\right)\right)$ the relaxation time could be obtained as 


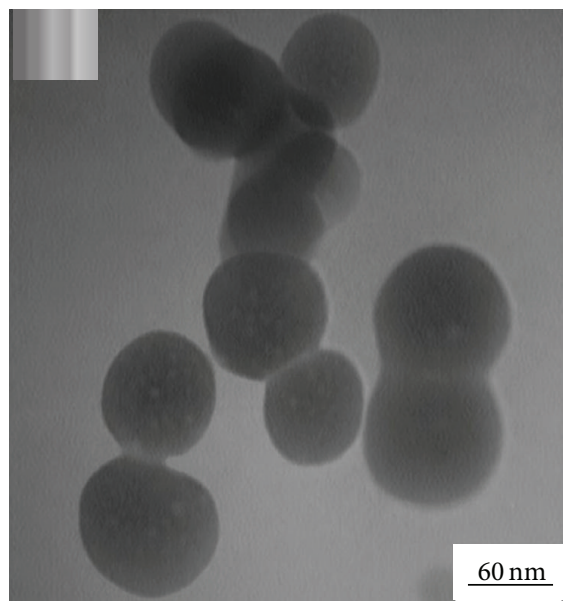

(a)

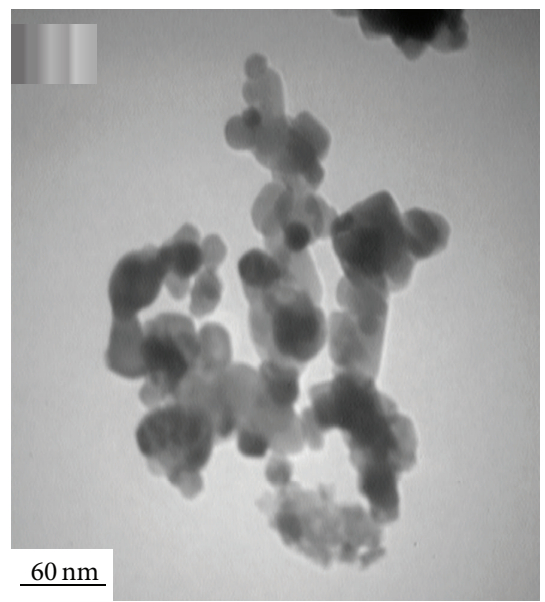

(b)

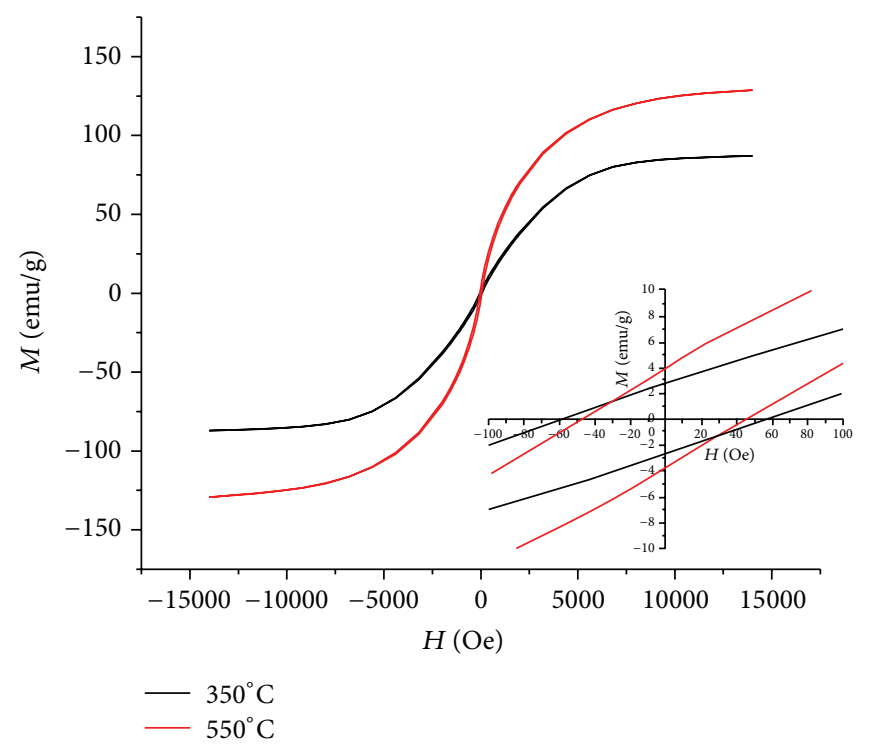

(c)

FIGURE 6: TEM images of W3 sample annealed for 20 minutes at (a) $550^{\circ} \mathrm{C}$ and (b) $350^{\circ} \mathrm{C}$. (c) Hysteresis curves for annealed samples.

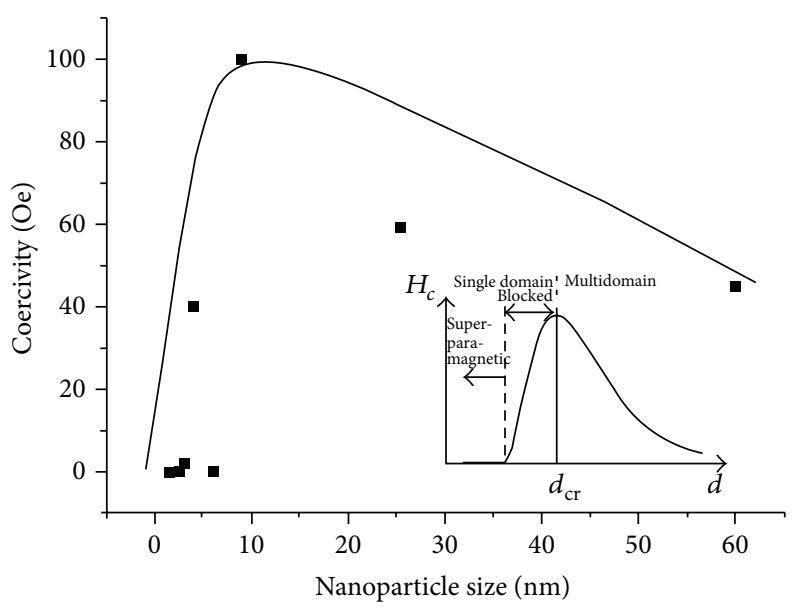

FIGURE 7: Coercivity as a function of particle size (continuous line is the trend, inset: [1]). 
$\tau_{R}=1.171 \times 10^{-9}(\mathrm{~s})$. Finally from (1), the area of the dynamic hysteresis loop would be $A=2.62 \times 10^{-8}(\mathrm{~mJ} / \mathrm{g})$ with the corresponding SAR $=0.262 \times 10^{-2}(\mathrm{~W} / \mathrm{g})$. This is a rough estimation for W3 sample because the anisotropy field was assumed to be equal to that of W4 sample. But qualitatively it could be deduced that for W4 sample in the single domainmulti domain boundary the SAR is much higher (about $10^{5}$ times) than W3 sample at ferromagnetic-superparamagnetic transition. Indeed the reason lies in the loss mechanism: hysteresis loss versus the Neel-Brown relaxation. The former is described by the Stoner-Wohlfarth models which assume null thermal activation and predict a square hysteresis area (W4 sample), but the latter assumes thermal equilibrium and a linear response of magnetic system to applied magnetic field (W3 sample).

Theoretically it is expected that the higher the frequency of the applied field the higher the losses. But experimental results indicate that by increasing the frequency of the applied field the generated heat reaches a frequency independent maximum. This could be due to nanoparticle interaction which makes hysteresis area independent of frequency [31]. In fact the above calculations are based on the hypothesis that the nanoparticles do not have considerable interactions with each other. In order to take the interactions into account, Monte-Carlo simulations should be used. But there is a severe problem in matching the time in simulations with the real time and to the best of the authors' knowledge there is no theoretical expression for this problem [31].

Another important point to be highlighted is that the above calculations neglect the Brownian relaxations (physical motion of nanoparticles under the influence of the magnetic field) in colloidal solution. By considering the effect of this type of relaxation some of the applied field will be dissipated due to the physical rotation of nanoparticles to align them along the applied field and the generated heat will be different from the above values.

Based on the large obtained theoretical SAR it could be inferred that W4 sample (with mean size of $9 \mathrm{~nm}$ ) has a great advantage to be used in magnetic hyperthermia treatment. But to assay these theoretical results which are based on the simple SW model, real experiments will be the subject of the future work to measure the values of A and SAR comparing them with these theoretical results.

\section{Conclusions}

Size controlled synthesis of FeCo nanoparticles was done using microemulsion method. The main parameters which determine the particle size are water to surfactant molar ratio $(R)$ and molar concentration of metal salts. By increasing the former the size of micelles increases leading to larger terminal nanoparticles and by raising the latter the number of formed nuclei increases leading to smaller terminal nanoparticles.

Size dependency of magnetic properties including $M_{s}$ and $H_{c}$ was investigated. The observed increase of $M_{s}$ with size is due to the disappearance of the magnetic dead layer in larger nanoparticles. But the observed change in coercivity is due to the transition between various size regimes and consequently the magnetization reversal mechanisms. Based on the variations of coercivity the superparamagneticferromagnetic and single domain-multi domain transition sizes were determined.

Then the magnetic losses were calculated at transition points based on the Stoner-Wohlfarth and LRT models. For W4 sample which is at the single domain-multi domain ferromagnetic transition point the anisotropy field, $H_{\mathcal{c},(T=0 \mathrm{~K})}$, and SAR were calculated using the Stoner-Wohlfarth model. The results are comparable to the highest reported in the literature. It should be noted that the results are applicable only when $x_{0} H_{\max } \geq x_{0} H_{c,(T=300 \mathrm{~K}, f=100 \mathrm{KHz})} \approx 70 \mathrm{mT}$.

But for W3 sample at superparamagnetic-single domain ferromagnetic transition the approximate SAR was obtained very lower than that of W4 sample. Based on the large obtained theoretical SAR it could be concluded that W4 sample (with mean size of $9 \mathrm{~nm}$ ) has a high potential to be used in magnetic hyperthermia treatment. Future experiments such as calorimetry could assay the theoretical results of the present research.

\section{Conflict of Interests}

The authors declare that there is no conflict of interests regarding the publication of this paper.

\section{References}

[1] S. P. Gubin, Y. A. Koksharov, G. B. Khomutov, and G. Y. Yurkov, "Magnetic nanoparticles: preparation, structure and properties," Russian Chemical Reviews, vol. 74, no. 6, pp. 489520, 2005.

[2] K. Zábranský, B. David, N. Pizúrová, O. Schneeweiss, R. Zbořil, and M. Mašláň, "Preparation and properties of FeCo nanoparticles," in Mossbauer Spectroscopy in Materials Science, vol. 1258 of AIP Conference Proceedings, pp. 102-107, 2010.

[3] D. Kodama, K. Shinoda, K. Sato, Y. Sato, B. Jeyadevan, and K. Tohji, "Synthesis of size-controlled Fe-Co alloy nanoparticles by modified polyol process," Journal of Magnetism and Magnetic Materials, vol. 310, no. 2, pp. 2396-2398, 2007.

[4] M. Abbas, M. NazrulIslam, B. P. Rao, T. Ogawa, M. Takahashi, and C. G. Kim, "One-pot synthesis of high magnetization air-stable FeCo nanoparticles by modified polyol method," Materials Letters, vol. 91, pp. 326-329, 2013.

[5] U. Cho, K. Wang, G. W. Kim, and B. H. Koo, "Effects of Ag seed on synthesis of FeCo nano-particles prepared via the polyol method," Journal of Materials Science and Technology, vol. 26, no. 7, pp. 660-664, 2010.

[6] G. Suresh, P. Saravanan, and D. R. Babu, "One-pot synthesis of Fe-Co nanospheres by modified polyol process and their structural, magnetic studies," Journal of Physics, vol. 292, no. 1, Article ID 012015, 2011.

[7] Q. Nguyen, C. N. Chinnasamy, S. D. Yoon et al., "Functionalization of FeCo alloy nanoparticles with highly dielectric amorphous oxide coatings," Journal of Applied Physics, vol. 103, no. 7, Article ID 07D532, 2008.

[8] S. J. Lee, J. H. Cho, C. Lee, J. Cho, Y. R. Kim, and J. K. Park, "Synthesis of highly magnetic graphite-encapsulated FeCo nanoparticles using a hydrothermal process," Nanotechnology, vol. 22, no. 37, Article ID 375603, 2011. 
[9] E. Holodelshikov, I. Perelshtein, and A. Gedanken, "Synthesis of air stable $\mathrm{FeCo} / \mathrm{C}$ alloy nanoparticles by decomposing a mixture of the corresponding metal-acetyl acetonates under their autogenic pressure," Inorganic Chemistry, vol. 50, no. 4, pp. 1288-1294, 2011.

[10] G. H. Lee, S. H. Huh, J. W. Jeong et al., "Structural and magnetic properties of bimetallic FeCo nanoclusters," Journal of the Korean Physical Society, vol. 42, no. 3, pp. 367-370, 2003.

[11] Z. Guo, L. L. Henry, and E. J. Podlaha, "CoFe, Fe and Co nanoparticles displacement with $\mathrm{Cu}$ ions," ECS Transactions, vol. 3, no. 25, pp. 337-345, 2007.

[12] X. W. Wei, G. X. Zhu, Y. J. Liu, Y. H. Ni, Y. Song, and Z. $\mathrm{Xu}$, "Large-scale controlled synthesis of FeCo nanocubes and microcages by wet chemistry," Chemistry of Materials, vol. 20, no. 19, pp. 6248-6253, 2008.

[13] S. J. Shin, Y. H. Kim, C. W. Kim, H. G. Cha, Y. J. Kim, and Y. S. Kang, "Preparation of magnetic FeCo nanoparticles by coprecipitation route," Current Applied Physics, vol. 7, no. 4, pp. 404-408, 2007.

[14] C. S. S. R. Kumar and F. Mohammad, "Magnetic nanomaterials for hyperthermia-based therapy and controlled drug delivery," Advanced Drug Delivery Reviews, vol. 63, no. 9, pp. 789-808, 2011.

[15] R. Hergt, S. Dutz, R. Müller, and M. Zeisberger, "Magnetic particle hyperthermia: nanoparticle magnetism and materials development for cancer therapy," Journal of Physics Condensed Matter, vol. 18, no. 38, pp. S2919-S2934, 2006.

[16] J. Carrey, B. Mehdaoui, and M. Respaud, "Simple models for dynamic hysteresis loop calculations of magnetic singledomain nanoparticles: application to magnetic hyperthermia optimization," Journal of Applied Physics, vol. 109, no. 8, Article ID 083921, 2011.

[17] M. Hesani, A. Yazdani, B. Abedi Ravan, and M. Ghazanfari, "The effect of particle size on the characteristics of FeCo nanoparticles," Solid State Communications, vol. 150, no. 13-14, pp. 594-597, 2010.

[18] A. N. Popova, Y. A. Zaharov, and V. M. Pugachev, "Chemical synthesis, structure and magnetic properties of nanocrystalline Fe-Co alloys," Materials Letters, vol. 74, pp. 173-175, 2012.

[19] C. Destrée and J. B. Nagy, "Mechanism of formation of inorganic and organic nanoparticles from microemulsions," Advances in Colloid and Interface Science, vol. 123-126, pp. 353-367, 2006.

[20] M. A. López-Quintela, "Synthesis of nanomaterials in microemulsions: formation mechanisms and growth control," Current Opinion in Colloid and Interface Science, vol. 8, no. 2, pp. 137-144, 2003.

[21] R. M. Espí, C. K. Weiss, and K. Landfester, "Inorganic nanoparticles prepared in miniemulsion," Current Opinion in Colloid and Interface Science, vol. 17, no. 4, pp. 212-224, 2012.

[22] F. J. Schork, Y. Luo, W. Smulders, J. P. Russum, A. Butté, and K. Fontenot, "Miniemulsion polymerization," Advances in Polymer Science, vol. 175, pp. 129-255, 2005.

[23] C. C. Wang, D. H. Chen, and T. C. Huang, "Synthesis of palladium nanoparticles in water-in-oil microemulsions," Colloids and Surfaces A, vol. 189, no. 1-3, pp. 145-154, 2001.

[24] C. Y. Wang, W. Q. Jiqng, Y. Zhou, Y. N. Wang, and Z. Y. Chen, "Synthesis of $\alpha$-Fe ultrafine particles in a saturated salt solution/isopropanol/PVP microemulsion and their structural characterization," Materials Research Bulletin, vol. 35, no. 1, pp. 53-58, 2000.
[25] M. A. López-Quintela, C. Tojo, M. C. Blanco, L. G. Rio, and J. R. Leis, "Microemulsion dynamics and reactions in microemulsions," Current Opinion in Colloid and Interface Science, vol. 9, no. 3-4, pp. 264-278, 2004.

[26] M. Ethayaraja and R. Bandyopadhyaya, "Population balance models and Monte Carlo simulation for nanoparticle formation in water-in-oil microemulsions: implications for CdS synthesis," Journal of the American Chemical Society, vol. 128, no. 51, pp. 17102-17113, 2006.

[27] J. T. Miller, A. J. Kropf, Y. Zha et al., "The effect of gold particle size on $\mathrm{Au}-\mathrm{Au}$ bond length and reactivity toward oxygen in supported catalysts," Journal of Catalysis, vol. 240, no. 2, pp. 222234, 2006.

[28] D. X. Chen, O. Pascu, A. Roig, and A. Sanchez, "Size analysis and magnetic structure of nickel nanoparticles," Journal of Magnetism and Magnetic Materials, vol. 322, no. 24, pp. 3834$3840,2010$.

[29] K. D. Bakoglidis, K. Simeonidis, D. Sakellari, G. Stefanou, and M. Angelakeris, "Size-dependent mechanisms in AC magnetic hyperthermia response of iron-oxide nanoparticles," IEEE Transactions on Magnetics, vol. 48, no. 4, pp. 1320-1323, 2012.

[30] G. Herzer, "Grain size dependence of coercivity and permeability in nanocrystalline ferromagnets," IEEE Transactions on Magnetics, vol. 26, no. 5, pp. 1397-1402, 1990.

[31] L. M. Lacroix, R. B. Malaki, J. Carrey et al., "Magnetic hyperthermia in single-domain monodisperse FeCo nanoparticles: evidences for Stoner-Wohlfarth behavior and large losses," Journal of Applied Physics, vol. 105, no. 2, Article ID 023911, 2009.

[32] B. Mehdaoui, A. Meffre, L. M. Lacroix et al., "Large specific absorption rates in the magnetic hyperthermia properties of metallic iron nanocubes," Journal of Magnetism and Magnetic Materials, vol. 322, no. 19, pp. L49-L52, 2010.

[33] X. Wang, H. Gu, and Z. Yang, "The heating effect of magnetic fluids in an alternating magnetic field," Journal of Magnetism and Magnetic Materials, vol. 293, no. 1, pp. 334-340, 2005. 

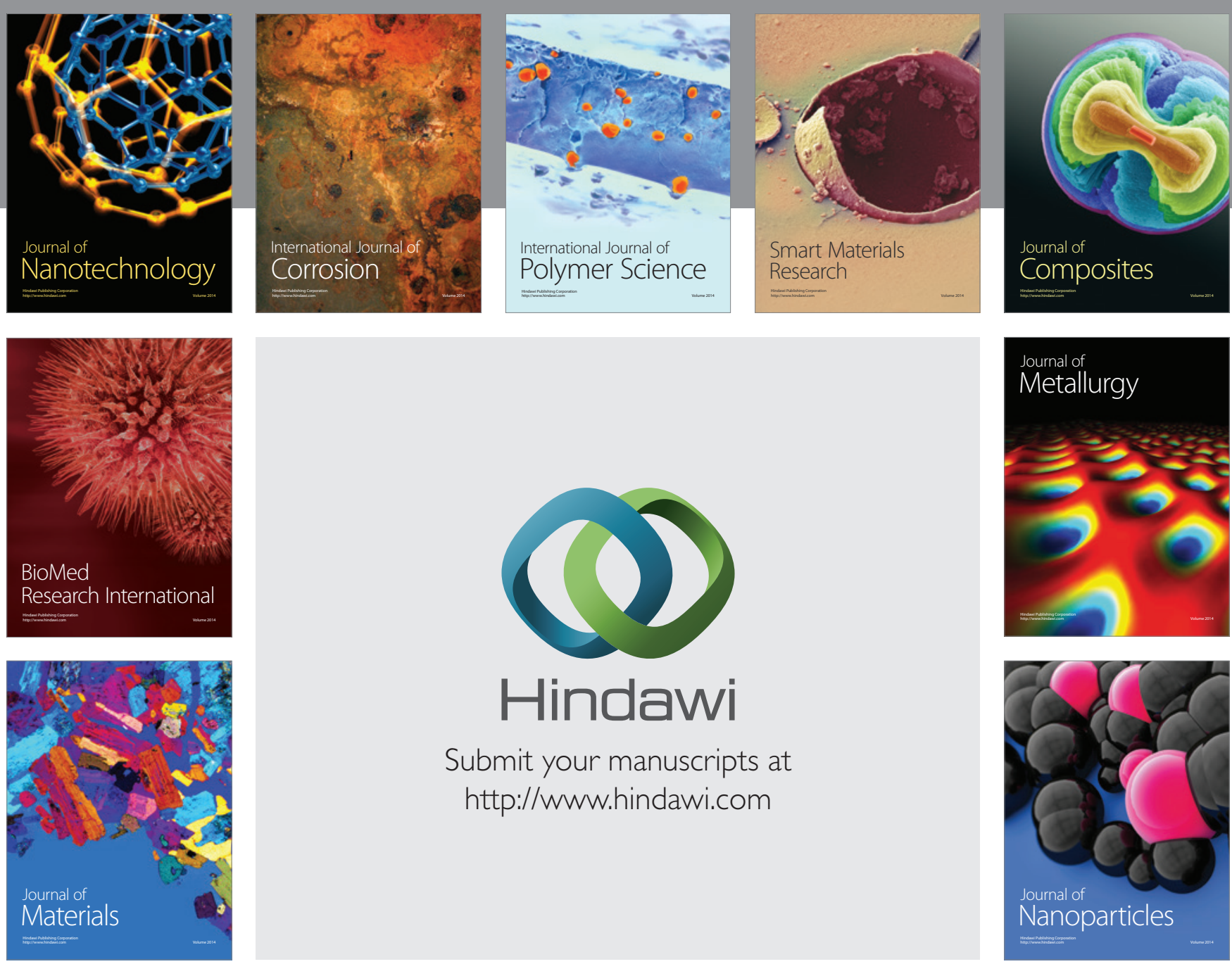

Submit your manuscripts at http://www.hindawi.com
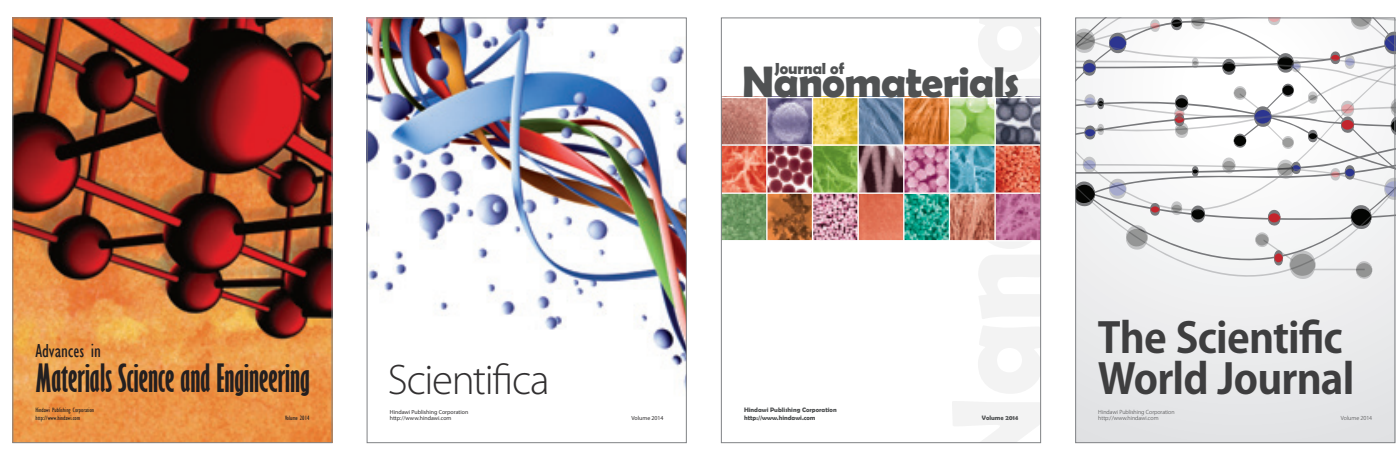

\section{The Scientific World Journal}
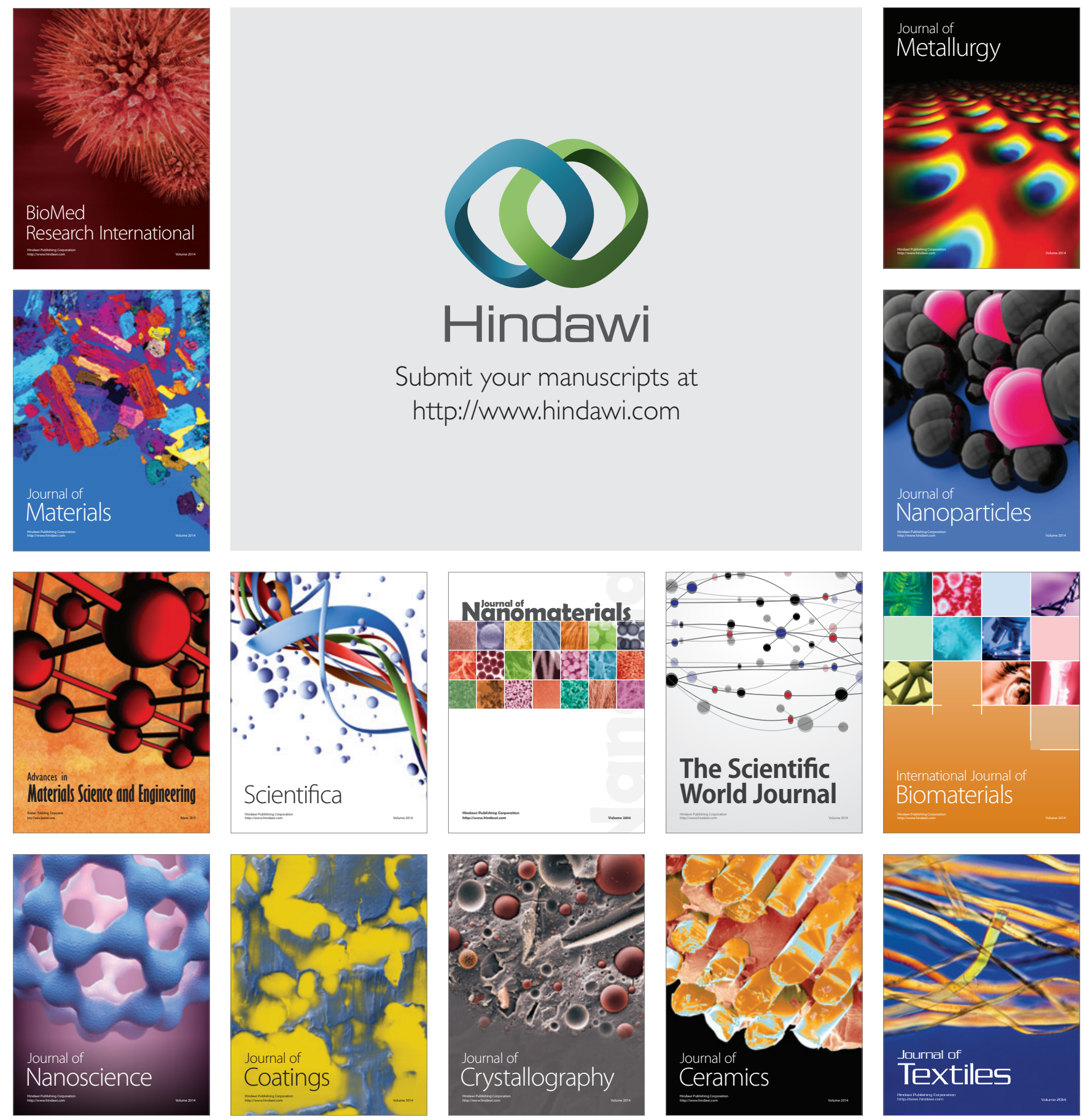